\title{
67. Microradiographic Subtraction Method combined with Histology for the Study of Interconnections between the Central Arteries of Human Brain
}

\author{
Toshisuke Yamamoto, Tomoo Kagami, Akio Yamagiwa \\ and Yoshifumi Kawarada \\ Surgical Department, Mie Prefecture University Medical School
}

Microradiographic subtraction method combined with histological examination was effectively applied to the research of vascular architecture of the central vessels. By the method all the suspected interarteriolar anastomoses were always proved to be only overpassings. While the network between capillaries were discriminated merely in histological preparations, those ascertained by the X-ray study alone were restricted to the pre- and postcapillary in nature. The precapillary rate was slender and exquisite, on the other hand postcapillary venous one was vigorous and tortuous.

In the cases of cerebral juxta-basal teleangiectasia, the networks in clinical angiography were analized and it was ascertained that the pathological networks consisted of two elements, one was slender and delicate, appearing even in earlier phase, and the other vigorous and tortuous, manifesting subsequently. The former was considered to be the collateral through physiological precapillary networks of the central arteries due to thrombotic occlusion of the internal carotid artery just in its junction with Willis' circle, and the latter was suggesting the overflow from collaterals to the corresponding venous channels.

\section{Discussion to 67.}

\section{So-called Cerebral Arterial Rete with Idiopathic Occlusive Arterial Disorders in the Main Trunks of the Cerebral Arteries: A report of an Autopsy Case}

\author{
Hajime Handa, Hiroshi KaJikawa, Kouzo Sato and Junkoh Yamashita \\ Department of Neurosurgery, K'yoto University Medical School \\ Michiko Tamegar and Hideyuki Haibara \\ 2nd Department of Pathology, Kyoto University Medical School
}

Characteristic vascular abnormality around the circle of Willis, that is, marked stenosis or occlusion of the carotid artery accompanied by "cluster" of the fine vessels, has been demonstrated in the carotid angiography especially of the Japanese 